\title{
DEUX EXPOSITIONS D'UN ÉVÊQUE FORTUNAT SUR L'ÉVANGILE.
}

$\mathrm{D}$ ANs un homéliaire assez singulier de la Bibliothèque municipale de Troyes on rencontre, non sans quelque surprise, deux développements exégétiques; tirés évidemment d'un commentaire du premier Évangile, et l'un et l'autre annoncés par ce titre insolite :

$\begin{array}{lll}\text { ITEM EXPOSITIO FORTVNATI EPISCOPI IN } & \text { IN } \\ \text { (al. EX) EODEM EVVANGELIO. } & \end{array}$

Qui peut être cet évêque Fortunat, auteur d'un commentaire de l'évangile? C'est la question qui se pose, et à laquelle il n'est pas malaisé de donner une réponse satisfaisante.

Du manuscrit lui-même, le Trecensis 653 (180 fol.), il n'y a pas grand' chose à dire. 11 remonte au XI $\mathrm{XI}^{\bullet}$ siècle $^{3}$; mais on ne sait pas où il a été copié. On sait seulement, grâce à une note ajoutée au bas de la première page ${ }^{2}$, qu'il fut donné vers la fin du XIVe siècle, en $137^{2}$ probablement 3 , par « le roi de France » Charles V à " la librairie commune des Prêcheurs du couvent de Troyes. Il passa ensuite dans la bibliothèque des Pithou, et de là dans celle de l'Oratoire de Troyes.

Sa composition est plus intéressante. Il représente, pour le fond, le recueil des homélies de Paul Diacre depuis le début de l'Avent jusqu'au quatrième Dimanche après l'Épiphanie; mais aux pièces de la rédaction carolingienne, dont fort peu sont omises 4 , il ajoute -... et c'est ce qui constitue son originalité - des \& expositions 》 de l'évangile empruntées, directement ou non, aux ouvrages des Pères. Le résultat est un volume assez considérable, comprenant au total cent-dix homélies ou sermons.

Nous avons par exemple pour le premier dimanche de l'Avent, dont la péricope est, au IX $\mathrm{IX}^{\mathrm{e}}$ siècle, celle de l'entrée de Jésus à

\footnotetext{
1. Sauf le premier cahier (fol. 1-8), refait au XIIe siècle.

2. Elle est rapportée par HARMantr, Catalogue général des manuscrits des bibliothè4ues prubliques des Départements (série in-4º). II, 1855, p. 274.

3. Vuir la notice, plus précise, du manuscrit 267 qui a la même provenance. ib., p. 132.

4. Cette série comprend régulièrement 64 pièces. Je suis l'analyse de F. WiEgaNd, exacte pour cette partie : Das Homiliarium Karls des Grossen, 1887, p. 17-30.
} 
EXPOSITIONS D'UN ÉVÊQUE FORTUNAT SUR L'ÉVANGILE. I6I

Jérusalem (MT. XXI, I : Cum appropinquassent Therosolimis et uenissent Bethphage) $\times$ : d'abord, l'homélie régulière désignée par Paul Diacre, un morceau du si ennuyeux Opus imperfectum in Matthaeum ( $\left(\mathrm{D}_{2}\right)^{2}$; puis in sermon de Maxime pour l'Avent, également fourni par Paul Diacre, mais qui devrait venir un peu plus loin ( $\left(\mathrm{D}_{4}\right)_{3} 3$; et enfin renouvelant le contexte traditionnel, quatre extraits de commentaires, relatifs à la péricope de l'entrée à Jérusalem d'après S. Mathieu :

"sermo beati Hylarii episcopi de eodem 》: Duo discipuli ad uicum missi sunt - meditatur operatio 4 ;

"expositio sancti Ieronimi presbyteri ex eodem euuangelio 》: Creditur's de Hierico turbis eductis - et infernorum. Prestante etc. ${ }^{6}$;

"expositio Fortunati episcopi in eodem euuangelio 》 ( $=$ fol. rov - I I v);

(omelia Origenis ex eodem euuangelio 》: Dignum est ostendere hic uoluntatem - dicebant osanna in excelsis deo q. u. et $r .7$

De même au lendemain de Noël, pour la fête de saint Étienne, nous avons les quatre pièces marquées par Paul Diacre (P D 27-30) ${ }^{8}$, dont la dernière est un extrait du commentaire de saint Jérôme sur la péricope de la fête (MT.XXIII, $34:$ Ecce ego mitto ad wos prophetas), ensuite quatre pièces de supplément :

un sermon mis sous le nom de saint Augustin : F. k. celebrauimus hesterna die natalem quo rex martyrum - et Stephanum gloriosim ad palmam martirii prouexit ecclesia. In X. I. dno n. ${ }^{9}$;

un extrait du commentaire de saint Hilaire: Iudicii forma in absoluto est - confessione benedicent to ;

« item expositio Fortunati episcopi ex eodem euuangelio $»(=$ fol. $94 \mathrm{v}-95 \mathrm{r})$

1. Elle a disparu dans la suite du moyen âge ot s'est trouvée réservée aux fonctions du dimanche des Rameaux; la première place fut ainsi laissée à l'annonce du jugement Mernier (LC. XXI, 25: Erunt signa in sole). A la fin du Xre siècle, lauteur du Miorologue retient encore l'usage ancien, tout en constatant l'usage nouvean (c. XXX:
$P . L$., CLI, 1003).

2. Puto res ipsa oxigit (P. A., LVI, 834 : homelie 37). 3. Igitur quoniam post tempus ( $P . \mathrm{L}$., LVII, 225 : homélie 2 [Mouet fortasse wos,]
cf. ib., note $i$ ).

4. Cf. P. L., IX, $1034 \mathrm{~B}-1036 \mathrm{~B} ; \mathrm{c} . \mathrm{XXY}$ § 1-3.

5. Lire : Egreditur.

6. Cf. $P . L$, XXVI (ed. 1884), 152A - 154C: in Mt. 1. III, c. XXI v. 1.9.

7. Cf. $P$. $G$., XIII, 1417-1438 (an bas des pages): Tome 16 (Vetus interpr.), \& 14-18.

8. Voir là-dessus WIEGAND, op. 1., p. 25.

9. Sermon inédit sous cette forme; on trouvera un doublet sous le to $\mathrm{CCXV}$ de $I^{\prime} A p p e n d i c e(P . L . X X X I X, 2145)$; d'autres rédactions se rencontrent, par exemple
dans le manuscrit 59 de Montpellier (s. X), f, 1200 v.

10. Cf. P. L., IX, $1050 \mathrm{D}-1052$ C : c. XXIV $\$ 8-11$. 
un extrait du commentaire d'Origène: Quantum ad primam faciem pertinet - quando fuerit quis conuersus ad eum qui $u$. et $r .{ }^{x}$

Tel est le contexte des deux « expositions $\gg$ qui nous intéressent. $J$ 'incline à croire que la présente compilation remonte au IX ${ }^{\circ}$ siècle et que son rédacteur a demandé à des recueils déjà formés et plus anciens tous les morceaux étrangers à l'homéliaire de Paul Diacre. En d'autres termes, il me semble raisonnable de supposer dès à présent que les développements de a Fortunat n'ont pas été tirés, au $\mathrm{IX}^{\mathrm{e}}$ siècle, d'un commentaire entier de cet auteur, conservé jusqu'à ce même IXe siècle, mais plutôt qu'ils survivaient déjà à part, depuis un temps qu'on ne saurait déterminer.

Lisons maintenant les textes. Ils sont réellement si simples que peu de remarques suffisent à les introduire.

L'auteur ne s'est pas mis en frais de style, ni même de réflexion. Il s'est contenté, le plus souvent, de jeter des notes à peine rédigées et mal raccordées 2, pour faire principalement ressortir l'allégorie de la lettre : spiritali intellectu (II, 1.27). Il n'était pas cependant incapable d'écrire, ni de traiter un sujet; témoin les passages parallèles sur le symbolisme de l'ânon que Jésus choisit pour monture (I, 1. 22-40), et sur les mystères de la poule, image des sollicitudes du Christ et de l'Église (II, 1. 27-44). Sans être très soignées, ces deux portions ne manquent pas de substance ni de mouvement ni d'une certaine précision verbale. De plus, la préoccupation morale s'y manifeste, derrière la recherche de l'allégorie; par ce souci, l'écrivain s'affirme bien un occidental, quelque but qu'il ait poursuivi en prenant la plume. Enfin, la Bible qu'il commente n'est pas la Vulgate de saint Jérôme, mais une version plus ancienne, de la famille dite européenne ou *italique 》, dans laquelle, autant qu'on en peut juger 3 , les leçons curieuses n'étaient pas rares.

1. Cf. $P$. G., XIII, $1630 \mathrm{~A}-1639 \mathrm{C}: \$ 25-28$.

2. Il est possible que, par endroits, notre manuscrit simplifie encore et même déforine plus ou moins l'original ; mais, tout moyen de contrôle faisant défaut, ce serait perdre son temps et sa peine que de rechercher et souligner les passages suspects. Ce qui no semble nullement vraisemblable, c'est une détérioration grave et systématique de lı matière livré. Ces textes, au total, ne doivent être ni pires ni meilleurs que la plupart des textes de l'antiquité et du haut moyen âge qui sont venus jusqu'à nous. J'ouerais même prétendre que leur apparence négligée est plutôt une garantie de la fidélití des organes transmetteurs.

3. C'e tit surtout à loccasion des textes bibliques que la rédaction du XIe siècle pourrait u'ètre pas très fidèle. Quelques passages paraissent pécher por omission; le plus sudvent au contrate, on a lien de craindre une assimilation à la rulgate. Par rapport aux ancions témoins. on notera en particulier ces cas daccord: XXI, I : appropin- 
Mais c'est surtout par un examen comparatif qu'on se rendra capable d'apprécier l'originalité et l'antiquité relatives de cette production littéraire, par elle-même d'un aspect un peu fuyant. Nous avons en effet, par bonheur, plusieurs séries exégétiques de la fin de la période antique ou du commencement du moyen-âge, qu'on peut mettre en regard des explications de « Fortunat 》: les annotations sur l'évangile ou Expositiunculae attribuées à Arnobe le Jeune ${ }^{\mathrm{x}}$; le commentaire de breui prouerbio du Pseudo-Jérôme ${ }^{2}$; les Allegories du Pseudo-Théophile ${ }^{3}$. 11 n'est que de parcourir ces maigres développements pour se bien convaincre de la supériorité de notre auteur; son exposé rapide garde une certaine saveur originale, qui fait habituellement défaut aux trois ouvrages mentionnés, même à celui d'Arnobe. La ressemblance tient principalement au parti-pris évident, chez tous ces amateurs de scholies, de ne s'arrêter qu'aux détails les plus frappants du texte sacré. Que si l'on prend ensuite pour point de comparaison les grands recueils de l'époque carolingienne, on ne saisira plus que des différences; Raban, Claude, Chrétien, le Ps.-Bède, Paschase lui-même, à ne citer que les commentateurs de Saint Mathieu, ont pour unique souci de reproduire fidèlement et complètement la pensée des " pères »; le centon prolixe est dès lors le plus sùr procédé, et souvent l'on ajoutera l'indication des sources.

\section{I}

Cum appropinquasset Iherosolimis et uenisset Bethfage. Bethfage: beth, ebraice domus; fage, grece manducare. Domus facile intelligitur ecclesia, in qua corpus Christi editur a fidelibus.

Tunc Iesus misit duos ex discipuis dicens. Ite in castellum quod contra uos est. Ostendebat Iesus quia Iherusalem aduersus apostolos erat factura post passionem saluatoris; ideo dixit: Castellum quod contra uos. Ideo auten castellum designabat quia Iherusalem destrui habebat a Romanis et redigi ut castellum; quod factum est a Vespasiano, qui destruxit Iherusalem et Iudeos triumphavit.

\footnotetext{
quasset (texte africain, Veronensis, Corbeiensis); uenisset (afric, ( or 1,$)_{1}: e^{\prime}$ disipulis (s. Hilaire, qui ajoute suis); XXI, 5 : pullum eius (afric.); XX1. 5 : manellum subinga.

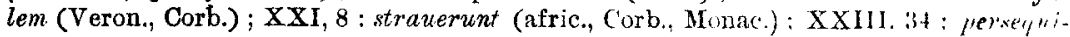
mini (Veron., Corb., Monac., Rhedig.) ; XXIII, 37 : notuistis (Veron., Corb.. Monake,); XXIII, 38 : relinquetur (la plupart des témoins anciens).

1. Cf. D. Monin, Études, textes, découvertes I, 1913, p. 34 ( $\mathrm{n}^{\circ} \mathrm{47}$ ).

2. Cf. D. G. WoHLenserg. Ein alter lateinischer Kommentar über die vier Eran. gelien, 1908. - Noter la juste remarque d'A. Souter. Journal of theological Studies, XIII, 1911, p. 155, à propos de cette compilation.

3. Cf, O. BARDENHEWER, Geschichte der allkirchlichen Literatur, I. 191:1, 1, 312 :s.
} 
Inuenietis asinam alligatam. Hoc est, synagogam dicit sub legis uinculo constitutam.

Et pullum eius cum ea. Soluite tt adducite mihi. Asina domita multis iam confractionibus populus extinctus inuenitur. Pullum autem solui et adduci : ostendit pullum populum nouellum. Quamuis enim quis senex sit cum crediderit, nouus efficitur. Pullum uero asinae solui, id est praedicatione apostolica factum ut omnia illa que erant grauia legis auferrentur; hoc est ergo soluere a legis uinculo liberare.

Pullum solutum ascendit Iesus : cui uestimenta supponuntur ut sederet Iesus. Est ergo pullus credens populus ex Iudeis quem nemo domauerat; id est, sepe missi prophetae duriciam eius populi edomare non ualuerunt. Vestimenta supposita mandata sunt quae per apostolos traduntur. Sedere autem super eum Iesum significatio est populum edoctum doctrina et mandatis apostolicis gestare super se saluatorem, qui $a b$ erroribus scilicet ido $<10>$ latriae retractus incipit sessorem thabere

30 filium dei. Sessor enim frenos habet ac tenet, quibus animal regit. Ergo habens populus christianus sessorem deum regitur frenis, id est disciplina et sanctitate et timore eius. Nam quod et calcaribus stimulatur est commonitio ne aut cespitet, aut non ita gradiatur ut decet, nec uelut pristino uicio adsuetus ad ullam lasciuiam redeat. Ideo ergo frenis frenatur, id est disciplina regitur, ut qui forte fuerat blasphemus iam ori suo custodiam imponat, et qui peruia lasciuus currebat in uiam rectam gradiatur, et qui forte per luxuriam cadere solebat iam firmus et stabilis ac fortis sit, ut possit 40 dominum quem gestat promereri.

Commemoratio sit etiam prophetiae : Ecce rex tuus uenit tibi mansuetus sedens super asinam et pullum nouellum subiugalem. - Asinam manifeste synagogam dicit. Pullum <nouellum> subiugalem : nouellum id est ex synagoga populum credentem futurum christianum; subiugalem autem propter traditam disciplinam legis, noui scilicet testamenti.

Plurima autem turbi strauerunt uestimenta sua iu uia. Spiritaliter uestimenta hic corpora ponit; in uia, id est in 50 mandatis dei quae apostoli tradiderunt. Denique ait: Et fecerunt discipuli sicut praecepit illis Iesus.

Ramos cedentes strauerunt in uia: in mandatis dei corpora sua, sed et filios sternere pro nomine dei deberent. Turbae autem praecedentes et quae sequebantur clamabant: Osanna filio David. Precedentes patres significant qui ante eum praedicauerunt; sequentes apostolos et ceteros ostendunt qui unica uoce dixerunt laudem filio David, id est Christo domino. 


\section{EXPOSITIONS D'UN ÉVÊUE FORTUNAT SUR L'ÉVANGiLe. I65}

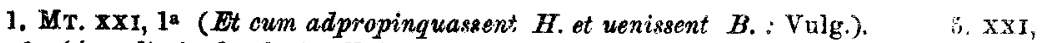

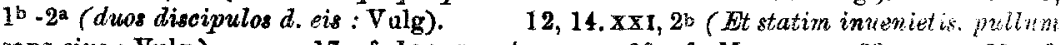

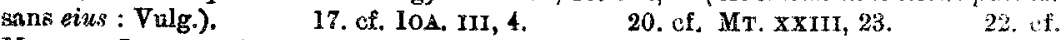

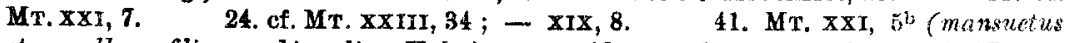
et; pullum filium subiugalis: Vulg.). 48. $\times \mathrm{XI}_{3} 8^{\mathrm{a}}$. 50. $\mathrm{XXI}, 6$ (Euntes autem discipuli fecerunt: Vulg.). $\quad$ 52. $\mathrm{XXI}, 8^{\text {b }}$ (alii autem caedebart ranus de arboribus et sternebant: Vulg). 54. $\mathbf{x x 1}, 9^{2}$ (quae praecedebant; clamabant dicentes: Vulg.).

1. Avant oum etc., le ms. donne: Secundum Matheum. In illo tempore; et après Bethfage : Et reliqua. 27. Il semble qu'un correcteur ail Ecrit Iesus. 27. Idw. latriae ms. 39. Il faudrait pent-être écrire : fit. 44. nouellum rétabii d'après la citation qui precède. 46. Peut-être legiz est-il de trop. 58, ms. ajoute: oui est honor et gloria in saecula amen.

\section{II}

Ecce ego mitto ad uos prophetas et sapientes et scribas et ex illis occidetis et crucifigetis, et persequimini de ciuitate in ciuitatem. Quod post passionem domini saluatoris impletum est. Nam Stephanum et Iacobum occiderunt, et apostolis uel ceteris discipulis multas et graues tribulationes inflixerunt carceribus recludendo et flagellando $\langle$ et $\rangle$ de ciuitatibus fugando. Completa sunt ergo quae ante praedixerat dominus facturos eos.

Hierusalem Iherusalem quae occidis prophetas. Increpat populum Iudaicum inhabitatorem ciuitatis eius, uidelicet ubi ius omne legis et forum.

Quotie $<n>s$ uolui : id est frequenter monui per prophetas et argui. Nouissime ueniens ipse non est susceptus. Imputat ergo huic ciuitati : Occidis, inquit, prophetas quos misi et lapidas, quotiens uolui congregare filios tuos.

Inpurat synagogae dicens : $\mathbf{S u b}$ alas. -- Sicut gallina pullos suos sub alas suas nutrit et fouet, sic uolui, inquit dominus, filios tuos congregare, sed noluisti.

Et quia noluisti $<s>$, relinquetur domus uestra deserta: deserti scilicet a spiritu sancto quem sepem in uineam posuit, tuitionem scilicet spiritalem. Domus autem deserta ipsos homines a spiritu sancto desertos atque derelictos accipit.

Quod autem dicit : Sub alas, alae accipiuntur Moses et Aaron, qui populum de Egipto eduxerunt, ut ait in cantico Deuteronomii : Extendit alas suas et suscepit eos. Alae autem nunc in ecclesia principes et apostoli sunt, qui doctrina sua ecclesiam protegunt. Gallina spiritali intellectu ecclesia accipitur. Sicut enim gallina oua etiam aliena quae non peperit cuiuslibet auis calefacit, aeducat ac nutrit, sic ecclesia ex omnibus nationibus venientes suscipit, et gratia sancti spiritus calefaciens ab alis suis, id est per doctrinam apostolicam - quae sunt alae ecclesiae, corda replens ac sacians cybo spiritali, el per unum spiritum 
et protectionem alarum efficit de uariis nationibus unum populum deo. Vt autem ecclesiam in gallinae figura cognoscas, sic figura facturae spei praecurrit. Denique intellige pulli sanguinem interfecti misceri cum aqua ac aspergi populum et sanctificari. Si ergo pullus cuius sanguine populus sanctificatur Christus intelligitur, qui pro ecclesia sanguinem fundens eam sanctificat, sine dubio gallina ecclesia accipi potest, sicut meminit Salomon : Catulus leonis - quod bene ingrediatur - et gallus ambulans inter gallinas laetus, id est inter ecclesias quas etiam Iohannes dicit septem candelabra.

1. MT. XXIII, $3 \pm$ (Ideo ecce; crucifigetis <et ex eis flagellabitis in synagogis uestris>

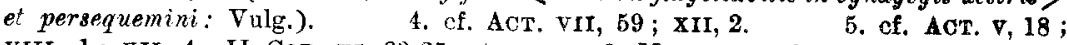
VIII, 1 ; XII, 4; II COR. XI, 23-25, etc. 9. MT. XXIII, 37a. $12(14,18)$. ib. $37^{\mathrm{b}}$ 13. cf. MT. $\mathrm{x} \times \mathrm{x}, 37$. 19, cf. MT. XXIm, 38 (noiuisti: Vulg.: relinquitur : leçon hiéronymienne d'après Wordsworth-White). 20. cf. Es. v, 2. 23. cf. EX. VI, 26, etc. 25. DEUT. XXIII, 11 (expandit; assumpsit eos: Vulg.). 37. cf. FX. XXIV, 6-8; LEV. XIV, 4-7; HEBR. IX, 19. 40. cf. ACT. XX, 28; ROM. V, 9 ; Apoc. I, 5, etc. 42. Prov. $\times x X, 30^{\mathrm{a}}[\mathrm{LXX}: \mathrm{XxIV}, 65]$ (Leo: Vulg.); les mots quf suivent, peut-être déplacés (voir ci-dessous), se réfèrent évidemment au verset 29 [LXX : 64] : tria sunt quas bene gradiuntur et quartum quod incedit feliciter, d'après Vulg. 43. $\quad$ XXX, $31[\mathrm{XXIV}, 66]$ (Gallus suecinetus lumbos:Vulg.). 44 . cf. APOC. I, 20.

2. Après erucifigetis; on peut supposer l'omission accidentelle du membre de phrase: et ex eis... uestris persequimini est bien la leçon de l'ancienne version latine. 6. Je rétablis et $2^{\circ}$. $\quad$ 12. quoties ms., mais cf. 1.15 . 19. noluisti ms, is correction noluistis, conforme à l'ancienne version latine, est indiqué par les mots nestra et deserti (et cf. 1. 22). 23, moyses de $2^{\circ}$ main. 25. deuteronomio ms. 29. Un correcteur a biffé aliena. 35. Il faudrait peut-être écrire: dei. 36. cognoscas est sur un grattage. 42. On peut supposer ici un déplacement, et restituer par exemple: meminit s. quod bene ingreditur: Catulus etc.; la référence an passage des Proverbes s'entendrait mieux. 44. En marge on lit cette note de $2^{\circ}$ main : septem aeclesias.

Ces morceaux étant ce qu'ils sont, la question d'auteur se pose d'une manière si précise, voire si pressante, qu'il teste peu de marge pour la discussion. Ce n'est pas souvent que l'histoire littéraire se pique de logique à ce point. Voici les termes entre lesquels, si je ne m'abuse, elle enferme le chercheur: il y n'a point de Fortunat à qui l'on puisse attribuer les deux expositions ; on connait au contraire un Fortunatien tout indiqué pour en recevoir le patronage.

Avant d'articuler mieux ces propositions un peu roides, il importe d'établir que la différence matérielle des noms ne crée aucune difficulté. Ils sont assez proches l'un de l'autre, évidemment, pour qu'un copiste qui n'y regardait pas de trop près ait pu les confondre. Mais à l'examen, les faits sont beaucoup plus significatifs. Des deux formes concurrentes Fortunatus _. Fortunatianus, la 
première est considérablement plus répandue dans l'ancienne Église latine, c'est-à-dire en Afrique et en Italie presque exclusivement . L'excellent répertoire de Smith et Wace signale, pour les huit premiers siècles, une soixantaine de Fortunats qui ont laissé une trace certaine dans l'histoire 2. D'ailleurs, ce relevé est encore incomplet, puisque le Martyrologe Hiéronymien énumère à lui seul quarantecinq Fortunatus, plus seize Fortunata 3 , dont la plupart sont autrement inconnus. Au même Martyrologe, chose curieuse, un seul Fortunatien paraît, un Africain marqué aux ides de juin, dont le nom n'a été épelé correctement que par le manuscrit de Berne 4. Mais, cette fois, c'est le Martyrologe Hiéronymien qui ne suffit plus à donner une image approchée de la réalité. Si l'on élargit l'enquête, le résultat donne quatorze noms bien attestés, avec cette particularité que tous nous maintiennent ou ramènent en Afrique : onze titulaires de sièges épiscopaux, catholiques ou donatistes $5,1 e$ prêtre de Thagaste mentionné dans la lettre LXXX de saint Augustin, le frère de sainte Victoire dont parlent les Actes des saints Saturninus et Dativus ${ }^{6}$; enfin l'évêque d'Aquilée, sur l'origine duquel saint Jérôme nous renseigne exactement : Fortunatianus natione $A$ fer... 7 On est donc autorisé à tenir le cognomen a Fortunatien p pour une forme non seulement dérivée ou secondaire, mais indigène, toujours plus ou moins dépaysée hors d'Afrique, et par suite exposée à perdre son contour.

Ce sont ces déformations qu'il est présentement intéressant de surprendre. J'en vais rapporter plusieurs exemples caractéristiques, parmi nombre d'autres probables que l'état des éditions ne permet pas de vérifier comme on le souhaiterait.

r. Le cas de Fortunatien, martyr d'Afrique, a déjà été indiqué. La compilation a hiéronymienne mentionne son nom au $I_{3}$ juin,

1. A part l'évêque de Poitiers, originaire des environs de Trévise, les listes épiscopales de Gaule ne fournissent aucun nom ; pour le reste, je ne remarque qu'un Fortunat dans l'ancienne Gaule, le martyr et diacre de Valence, compagnon de saint Felix (23 avril).

2. A Dictionary of Christian Biography, II, 1880, p. 551 ss. (tenir compte des articles qui groupent plusieurs personnages). Au total, les Africains dominent ; l'apparence est que le nom s'est répandu d'Afrique en Italie.

3. Voir l'index de l'édition Rossi-DuchesNe, 1894, p. [168]; je totalise les nentions Fortunatus et Furtunatus, Fortunata et Furtunatu. Figurent aussi au Martyrolige : anze Fortunus, trois Fortuna et cinq Fortunio.

4. Ib., p. [78]; pour le détail, voir plus loin.

5. Ici les études du P. J. MesnaGa permettent un contrôle presque rigoureux: L'Afrique chrétienne, 1912, p. 579. - Peut-être faudrait-il ajouter. sil se distingue réellement de l'un ou l'autre des onze titulaires, le fortunatianus qui prit part an synode d'Hippone pour le choix d'Héraclius (n'9 de smiTH-WACE. op. l., p. 5j1).

6. Cf. RUINART, Acta sincera, 1689, p. 412 et 418 .

7. De uiris inlustribus, c. XCVII. 
dans un groupe où il occupe la seconde place. Le Bernensis est seul a donner : Furturiatiani ; les martyrologes d'Epternach et de Wissembourg disent au contraire : Furtunati . Maintenant qu'on a le témoignage du martyrologe de Ricemarch ${ }^{2}$, conforme sur ce point à celui de Berne, il n'y a aucun doute que la graphie traditionnelle ait été « Fortunatien ». C'est un des nombreux passages du Hiéronymien où l'important «bréviaire 》 de Richemarch permet de départager les familles rivales et de fixer la teneur première du texte 3 .

2. Le souvenir d'un autre Fortunatien, compagnon de saint Félix de Thibiuca, est consigné dans les martyrologes «historiques" au 30 septembre. Bède, Florus, Adon l'appellent d'accord \& Fortunatien » et sont en cela fidèles à l'ancienne Passion de saint Félix 4. Néanmoins, le principal exemplaire du Petit Romain, un manuscrit de Saint-Gall du Xe siècle, écrit : Fortunati ${ }^{5}$.

3. Parmi les onze Fortunatiens que signalent les listes épiscopales d'Afrique, on remarque un évêque de Neapolis (Proconsulaire), connu pour avoir assisté aux conciles de Carthage de 4 II et de 4I g. Le P. Mesnage a constaté que, sur dix manuscrits renfermant les actes de l'assemblée de $4 \mathrm{II}$, cinq dénommaient simplement ce personnage \& Fortunatus 6 .

7. Fortunatien d'Assuras est un évêque lapssus, contemporain de saint Cyprien. Son nom est rappelé dans la collection des lettres de l'évêque de Carthage $(E p . L X V)$. Deux manuscrits de cette collection, $C$ et $R$ du IX siècle, donnent à lire fautivement: Fortunatum 7 .

5. Voici un dernier fait plus intéressant et qui nous ramène directement à notre sujet. Claude de Turin, énumérant dans la préface de son ouvrage sur S. Mathieu les principaux commentateurs de l'évangile, inscrit parmi eux un \& Fortunat $\$$ 8. Or Claude dépend uniquement ici d'un passage célèbre de saint Jérôme 9 , tout de

1. Voir l'édition Rossi-Duchesne, l, c.

2. Cf. H. J. LAwlon, The Psalter and Martyrotogy of Ricemarch, I, 1914, p. 16. On peut comparer le texte établi par le P. Delemaye, Analecta Bollandiana, XXXII, 1913, p. 395.

3. Cf. LAWLOR, ib., p. XXV et suiv.

4. Cf. D. H. QuentiN, Les Martyrologes Historiqueg du moyen âge, 1908, p. 74, 454, 523 .

5. Ib., p. 444.

6. L'Afrique chrétienne: évêchés ot ruines antiques, 1912, p. 124, et cf. p. VII.

7. C. Cypriani opera, éd. HaRTEL, III, 2, 1871, p. 721, 16.

8. Voir ledition de Duemmler, Epistolae karolini aeui, II, 1896, p. 594, 29. Cf. $P$. L., CIV, 836 .

9. Comm, in ev. Mt., Prologus : P. L, XXVi (ed. 1881), 20 (Vallarsi, VII, 7). 
même que Raban Maur et Paschase Radbert dans leurs commen. taires parallèles à celui de Claude ${ }^{\mathrm{I}}$; mais Raban et Paschase, plus attentifs, ont reproduit correctement le nom de l'évêque d'Aquilée.

Avec Claude et Raban, nous touchons à un point délicat, ou qui du moins parait tel. Ces auteurs prétendent avoir lu et certifient qu'ils vont mettre en cuvre et citer largement non seulement saint Jérôme, mais divers a saints pères 》 ou (docteurs 》, depuis les plus anciens jusqu'à Bède. Ils ne donnent pas d'ailleurs la même liste ${ }^{2}$; mais ils proposent l'un et l'autre le nom de Fortunatien ( ( Fortunat 》 chez Claude), de telle façon qu'un lecteur non prévenu s'attend à trouver en leurs ouvrages des extraits de cet écrivain. En réalité, comme nous allons voir, leur manière de dire est ambiguë ; à défaut des références marginales, heureusement préservées dans les manuscrits et qui permettent de contrôler assez exactement leur promesse, il y aurait lieu d'hésiter sur le nombre et sur la qualité des sources auxquelles ils ont puisé. Paschase, au contraire, s'exprime fort nettenient: il sait - c'est-à-dire d'après saint Jérôme - que Victorin et Fortunatien ont publié une explication de S. Mathieu; mais il n'a point réussi à se procurer ces commentaires: " licet Fortunatianum et Victorinum in eo opuscula edidisse dicatur, quos neçum inuenire potuimus ». C'est précisément ce que nous attendions.

Claude indique en premier lieu douze écrivains, ses prédécesseurs, aux « traités $\triangleright$ desquels il a demandé son information. La série va d'Origène à Bède, en passant par saint Ambroise, Rufin, saint Léon; saint Augustin est de nouveau mentionné à part comme l'interprète le plus important inter omnes. Il est donc clair qu'en ce passage Claude n'a pas entendu prendre seulement pour répondants les Pères qui avaient expliqué didactiquement $\mathrm{S}$. Mathieu ou un autre Évangile, mais qu'il a établi une liste compréhensive de ses auteurs

1. Cf. Duemmler, op. l., III, 1899. p. 389, 1. 1 et $2 \delta$; et IV, 1, 1902, p. 138, 35. On trouvera ces mêmes textes dans MIGNE, P. L., CVII, 728, 729, et CXX, 34.

2. Je laisse de côté la question, insuffisamment ellueidée, des relations de Claude qui Ecrit en 815 et de Raban qui écrit en $82 \mathrm{~L}$; il nous faudrait, pour la bien régler, une Edition du commentaire de Claude, Schönbach a cru remarquer que Raban reprenait à son compte le travail de Claude (voir les Sitzungsberiohte de YAcalémie de Vienne, t. CXLV1, 1903, Abh. IV, p. 91 ss.), Hablitzel imagine une hypothèse différente (cf. Historisches Jahrbuch, XXVII, 1906, p. 74 ss.) : Raban dépendrait du Ps. Bède, qu'il citerait comme « Bède 》. Les sondages pratiqués par Schönbach sont fort adroits; il aurait fallu les étendre, pour créer une conviction. Hablitzel a pris beaucoup de peine pour analyser l'ourrage de Raban (cf. Hrabanus Maurus, 1906, p. 21-70); malheureusement, il s'est appuyé sur l'édition de Cologne, au lieu de recourir aux manuscrits, dont l'examen s'imposait. 
préférés. Tout aussitôt, Claude s'excuse d'avoir entrepris quast aliqua temeritate une ceuvre nouvelle, après celle de saint Jérôme, après celles de saint Hilaire et de a Fortunat $>$; et il s'excuse habilement en renvoyant à la lecture de ces mêmes commentateurs. Car il n'est pas moins clair maintenant qu'il s'agit des commentaires de S. Mathieu qui ont précédé le sien propre. Hilaire et Jérôme étaient déjà nommés parmi les douze Pères; de Fortunat seul, il n'avait pas été question, et pour cause. Quant à Victorin, il est complètement oublié.

Tout ceci se tient fort bien. J'ai fait la contrépreuve en parcourant les pages encore inédites de Claude $x$. Il se trouve qu'en effet douze sigles se présentent à tour de rôle dans les marges, deux ou trois sans doute exceptionnellement comme MAX., OR. et FVLG., mais un, plus fréquemment que tous les autres : IER. Ce sont tous les auteurs énumérés dans la lettre à l'abbé de Charroux, et l'on en chercherait vainement un treizième. «Fortunat n'était pour Claude qu'un souvenir littéraire.

Raban a procédé un peu différemment, d'après les confidences de sa lettre à Haistulphe. Il y rapporte en propres termes le texte de saint Jérôme relatif aux commentaires du premier Évangile. Il sait ainsi qu'indépendamment du bref ouvrage de saint Jérôme trois commentaires latins avaient seuls existé : ceux de saint Hilaire, de Victorin et de Fortunatien. C'était une raison pour en composer un nouveau ${ }^{2}$, à savoir : \& tam plenam et sufficientem expositionem... sicut in caeteros euangelistas 3. Raban nomme aussitôt saint Ambroise, saint Augustin et Bède ; et nous sommes en mesure de pénétrer son dessein : il s'est proposé un travail étendu, qui se puisse comparer, sous ce rapport, aux développements de saint Ambroise sur S. Luc, de saint Augustin sur S. Jean, de Bède sur $\mathrm{S}$. Marc et sur $\mathrm{S}$. Luc ${ }^{3}$. Après les excuses de rigueur,

1. Je me suis servi de deux manuscrits du British Museum, Royal $2 C X$ et $4 C V I I$, l'un et l'autre du XII' siècle. Dümmler en a noté une diraine (voir sa notice Ueber Leben und Lehre des Bischofs Claudius von Turin, dans les Sitzungsberichte de l'Académie de Berlin, 1895, p. 427 et suiv.; et cf. les Epistolae karolini aeui II, 1896,p. 593). Il y aurait encore beaucoup à faire pour mieux connaître l'œuvre de Claude.

2. De ceci il paraît bien résulter, à première vue, que Raban n'avait pas connaissance de l'onvrage de Claude, publié cinq ou six ans plus tôt. Schönbach se tire pourtant de cette difficulté en supposant que Raban s'était mis à l'œuvre et avait réuni la plupart de ses « autorités o avant d'avoir en mains la Catena de l'évêque de Turin ; et de fait, il semblerait que l'influence de celle-ci n'est pas sensible dans les premiers chapitres de la rédaction de Raban.

3. Il est curieux que le commentaire d'Alcuin sur $\mathrm{S}$. Jean ne soit pas rappele; car, en définitive, Raban comblait tout juste pour ses contemporains la lacune qui subsistait après les travaux de Bede et d'Alcuin sur les Évangiles. 
Raban poursuit en expliquant sa méthode et l'arrangement de ses extraits. Ici, comme l'a fait voir Schönbach, il ne fait que démarquer la lettre-préface du commentaire de Bède sur S. Luc pour Acca x. Mais là où Bède se réclame seulement des quatre grands docteurs de l'Église latine dont il harmonisera les citations, l'érudit Raban, grand dévoreur de livres, et d'ailleurs maître d'une riche bibliothèque, se perd dans une imposante énumération de noms qui ne laisse pas d'être suspecte ${ }^{2}$ :

Adgregatis igitur hinc inde insignissimis sacrae lectionis atque dignissimis artificibus, quid in opusculis suis in beati Mathei uerbis senserint, quid dixerint, diligentius inspicere curaui :

Cyprianum dico atque Eusebium, Hilarium, Ambrosium, Hieronimum, Augustinum, Fulgentium, Victorinum, Fortunatianum, Orosium, Leonem, Gregorium Nazanzenum, Gregorium papam romanum, Ioannemque Crisostomum et ceteros patres quorum nomina sunt scripta in libro uitae.

Comme pour Claude, il est aisé de vérifier l'exactitude de ce programme, puisque Raban a suivi également le système, introduit par Bède, des références expresses e latere. Nous avons cette fois l'avantage d'une édition, qui, à la différence de celles de Bède, n'a pas omis de reproduire les signa nominum, spécialement recommandés aux copistes ${ }^{3}$. Il n'en serait pas moins utile de se reporter aux manuscrits, aux plus anciens surtout ${ }^{4}$, s'il fallait faire une étude complète des citations de Raban ; à plus forte raison, si l'on voulait interpréter correctement les références annoncées et se préserver de trop grosses méprises. Les sigles ou symboles étaient un expédient commode et sont pour nous fort instructifs ; mais, frêles par eux-mêmes et parfois indistincts, ils ont été déformés, déplacés, oubliés volontairement ou non d'une copie à l'autre, et plusieurs dont la forme est certaine demeurent inintelligibles faute d'une table d'équivalence ${ }^{5}$.

1. Of. $P$. $L$, XC1I, 304 et 305 . - Voir ScBornbach, dissertation cité, p. 97 et suiv.

2. Les mots en italique sont repris de la lettre de Bède á Acca.

8. Multamque obsecro et per Dominum legentes obtestor nt, si qui forte nostra haec qualiacumque sunt opuscula transcriptione digna duxerint, memorata quoque minimum signa, ut in nostro exemplari reperiunt, affigere meminerint. 》 Cest la prière de Bede A Acca (1. c., 305 A), reprise sans l'omission d'un geul mot par Raban (cf. P. L. CVII, 729 C).

4. Dümmler en a indiqué cinq (Bpistolae karolini aeui, III, p. 388) ; $0^{\prime}$ est un nombre qu'on pourrait aisément tripler. J'ai pu consulter le manuscrit Harley 3104 du British Museum; il provient de Baint-Sauveur-le-Vicomte (diocesse de Contances) et remonte au XII siecle.

5. Voir par exemple, pour la série qui accompagne l'Expositio comitis de Smaragde, les observations d'A. SoUTER, Journal of theological Studies, IX, 1908, p. 586 et suiv. - 
Si l'on examine l'édition du commentaire de Raban ${ }^{x}$, on a la surprise d'y rencontrer une vingtaine de passages, ainsi désignés : -Venant. »Le premier se présente au livre $V$, à propos de Mr. XV, $3^{6}{ }^{2}$. Ils se multiplient au cours des derniers livres, c'està-dire VII et VIII 3. De Venance, nous n'en connaissons súrement qu'un seul : Venantius Honorius Clementianus Fortunatus, l'ami et biographe de sainte Radegonde, le précieux auteur des Carmina, venu d'Italie en Gaule en la seconde moitié du VI ${ }^{\mathrm{e}}$ siècle (vers 565) et mort évêque de Poitiers dans les premières années du siècle suivant. On n'a jamais oui dire qu'il ait écrit sur l'évangile, et nous nous doutons aussi bien de quel style il l'eût fait. L'hypothèse n'a pas même besoin d'être discutée, ni celle qui consisterait à attribuer au même personnage les deux expositions du manuscrit de Troyes. Comment expliquer donc cette étrange référence, sinon en supposant que celui qui l'a inscrite de parti-pris 4 plus de vingt fois en marge du recueil de. Raban s'est trouvé en présence d'un sigle obscur, et qu'il a tenté de l'identifier coûte que coûte avec l'un des noms mis en avant dans la lettre à Haistulphe ? C'était encore le cas de pratiquer l'échange Fortunatien - Fortunat. En désespoir de cause, et tout comme dans une série algébrique, on passait de Fortunatien à Fortunat, puis à Venance, afin de rejoindre la forme abrégée $V$ ou $V e n$ des marges de Raban. Ces divers passages en effet, dénotés «Venant.», ne sont rien autre chose, si l'on prend la peine de rechercher leur vrai contexte, que des extraits de l'ouvrage de Bède sur S. Luc. Dès lors, toute difficulté s'évanouit, et c'est bien $V$ ou Ven qu'a écrit Raban, mais pour signifier le Vénérable : Beda Venerabilis 5.

Ces explications sont confirmées par un autre détail isolé, mais

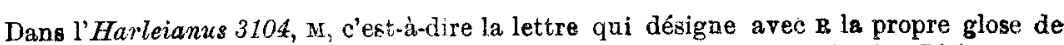
Raban, est régulièrement rendu par le groupe $\mathrm{MAX}_{\text {, }}$ et les emprunts à saint Jéôme sont indiqués : HAM.

1. Préparée par Pamelins et publiée par Colvenerius, Cologne 1625: t. V des Opera, p. 5-160 ; les renvois aux sourcé, sont dounes dans le cadre des marges. La réimpression de Migne, $P$. L. CVII,729-1156, a l'inconvénient d'avoir fait passer ces indications en plein texte, généralemeut après la portion du verset commenté. Je rappelle que le texte des quatre passages mutilés dans l'édition de Cologne (cf. P. L., ib., 1077B : le plus considérable, dans l' Harleianus, f. $121 \mathrm{v}^{\circ}$ à $127 \mathrm{r}^{\circ} ; 1096 \mathrm{c} ; 1099 \mathrm{c} ; 1148 \mathrm{D}$ ) a été publié par F. Kunstmans, Hrabanus Magnontius Maurus, 1841, p, 170-210.

2. P. L., ib., 984 A.

3. Ib., $1065 \mathrm{C}-\mathrm{D}, 1068 \mathrm{C}-\mathrm{D}, 1072 \mathrm{D}, 1074 \mathrm{~A}, 1079 \mathrm{C}$ et $\mathrm{D} ; 1124 \mathrm{~B}, 1125 \mathrm{C}, 1130 \mathrm{~A}$, $1132 \mathrm{D}, 1134 \mathrm{~B}, 1138 \mathrm{c}, 1139 \mathrm{C}, 1140 \mathrm{~B}, 1143 \mathrm{c}$.

4. L'éditeur de Raban pourrait être, à la rigueur, responsable de cette transcription; mais il est plus vraisemblable de l'attribuer au copiste même du manuscrit employé pour l'édition.

5. Ce qui ne l'a pas empêché de dire aussi souvent $\mathrm{B}=$ Beda. 
non moins bizarre, de l'édition de Cologne. On lit vers le début du livre III * un morceau étiqueté « Victorinus ». Au vrai, il s'agit encore d'une simple citation du même ouvrage de Bède 2. Mais ce qu'il faut remarquer, c'est qu'on a songé cette fois, et cette unique fois, à justifier par une fausse interprétation du sigle $V$ la présence du nom de Victorin parmi les sources annoncées de Raban.

Enfin, et ceci achèvera de mettre en évidence le caractère de ces bévues, j'ai pu constater que dans le manuscrit Harley 3104 tous les passages de Bède, dont il vient d'être question d'après le texte imprimé sont connotés, non plus "Venant. 》, mais «Vict. \# ou *Victor. *. Ce genre d'erreurs remonte donc assez haut, de même que le souci de retrouver au long des marges les auteurs inexistants dont Raban avait eu le tort de promettre des extraits.

Trompé par sa mémoire d'érudit, Raban avait été en effet imprudent en mentionnant, à l'exemple de saint Jérôme, Victorin et Fortunatien, comme s'il les devait citer. Pour le reste, il a bien tenu ses promesses et au delà ; ou du moins il n'enchérissait pas en indiquant qu'il puiserait aux écrits d'autres Pères que ceux énumérés. Le manuscrit que j'ai consulté présente, outre les noms atten-

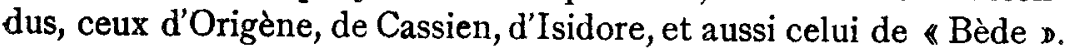

L'étude des commentaires du moyen-âge nous a pu convaincre qu'en dépit d'assurances frivoles ni l'ouvrage de Fortunatien ni celui de Victorin n'étaient parvenus, en tout cas dans leur intégrité, jusqu'aux exégètes du IXe siècle. Que des extraits en aient été retenus par hasard, tels que ceux du manuscrit de Troyes ${ }^{3}$, c'est là un phénomène tout différent, et que d'ailleurs l'on n'a pas à expliquer, mais seulement à enregistrer. L'édition du commentaire de Raban nous a donné l'occasion d'écarter sans plus le nom de Fortunat, évêque de Poitiers. Il n'y a désormais, après ce circuit, qu'à énoncer les titres de Fortunatien l'Africain, évêque

1. P. $L$., CVII, $855 \mathrm{D}-856 \mathrm{A-B}$.

2. P. L., XCII, 145 A-B.

3. Un détail propre au commentaire de Paschase (P. $L$, CXX, 791, B) sur Moïse et Aaron comparés aux ailes de la poule (MT. xxIII, 37) pourrait donner à croire que Paschase a connu les expositions du manuscrit de Troyes, on du moins la deuxieme (voir ci-dessus II, 1. 23). Mais on a dejà cette interpretation dans le Ps.-Chrysostome latin (P. G., LVI, 895.) Il est plus probable qu'elle était devenue nn lieu commun, bien que les principaux exégètes n'en parlent pas, a ma connaissance. Je l'ai d'ailleurs notée aussi chez les homélistes, jusqu'a présent si négligés, do l'école d'Auxerre: Haimon (P. $L .$, CXVIII, 69 B) et Heiric $(P . L ., \mathrm{XOV}, 1172 \mathrm{~B})$. 
d'Aquilée au temps de l'empereur Constance (337-36I) et du pape Libère $(352-366)$.

Saint Jérôme nous dit de lui dans la notice précédemment mentionnée :

Fortunatianus... in euangelia titulis ordinatis breui et rustico sermone scripsit commentarios.

II compare ailleurs ces commentaires à la perle précieuse de l'Évangile et les qualifie par la phrase du Psalmiste : «paroles du Seigneur, paroles chastes, argent éprouvé par le feu, sept fois raffiné ${ }^{2}$. Enfin, comme on l'a vu, il s'y réfère expressément dans son prologue sur S. Mathieu.

Nous n'avons pas d'autres renseignements sur l'ouvrage de Fortunatien. On a songé, bien à tort, à l'identifier avec le Ps.-Jérôme de breui prouerbio 3 . On a pensé aussi que Chromatius, titulaire du siège d'Aquilée à la fin du IVe siècle, s'était peut-être inspiré des remarques de son prédécesseur dans une suite de Tractatus sur S. Mathieu ${ }^{4}$ : hypothèse plausible, mais invérifiable. On possède du moins maintenant deux morceaux, pourvus d'une attestation littéraire très suffisante et qui répondent assez bien à la description de saint Jérôme.

D. A. WiLMART.

\footnotetext{
1. Sur sá part dans la rédaction des lettres du pape Libère, d'après saint Hilaire et saint Jerôme, voir L. Duchesne, Libère et Fortwnatien, 1908, et cf. Revue Bónédiotine, $\mathrm{XXV}, 1908, \mathrm{p} .360 \mathrm{ss}$.

2. Épist. X, 3 à Paul de Concordia (ed. Hrlbera, 1910, p. 37, 17 ss.)

3. Cf. WoH Lmangra. Ein alter lateinischer Kommentar über die viev Evangelien 1908, p. 29-36.

4. Cf. PAsCHini, Rerue Bénédictine, XXVI, 1909, 469, ss.
} 